der Dispositive und ihre Machtwirkungen - z.B. des Sexualitätsdispositivs umfassend beschrieben und damit die ersten analytischen Schritte dieses Forschungsstils unternommen. Auch der Präventionsdispositiv ließe sich daher mithilfe von Foucaults Machtanalytik beschreiben und auf seine Machteffekte hinterfragen.

Im Folgenden werden die Grundzüge von Foucaults Analytik vorgestellt, angefangen mit den theoretischen Abgrenzungen einer Geschichte der Problematisierungen, über die Beschreibung seiner drei analytischen Achsen - Wissen, Macht und Subjekt - bis hin zur Diskussion seines Konzepts der Gouvernementalität und des damit zusammenhängenden Verständnisses des Politischen, welche das Analyseverfahren der Dispositivanalyse begleiten werden.

\title{
1 Geschichte des Denkens als Analyse der Problematisierungen
}

Während der Entwicklung seiner Analyseinstrumente hat Foucault mehrere methodische und methodologische Umkehrungen und Abgrenzungen vorgenommen. Der Aufbau seiner Vorgehensweise ließe sich am deutlichsten anhand des Abschnitts seiner 1982/1983 gehaltenen Vorlesungen beobachten:

»In dem umfassenden Projekt, das unter dem Zeichen, wenn nicht gar unter der Überschrift einer >Geschichte des Denkens steht, sah ich mein Problem darin, etwas zu tun, das sich ein bisschen von dem unterscheidet, was die meisten Ideengeschichtler völlig zu Recht praktizieren. Jedenfalls wollte ich mich durch zwei Methoden absetzen, die beide übrigens ebenfalls völlig legitim sind. Zunächst wollte ich mich abgrenzen gegen das, was man Mentalitätsgeschichte nennen könnte und was tatsächlich auch so genannt wird. Diese Ceschichte erstreckt sich, schematisch betrachtet, von der Verhaltensanalyse bis zu den Äußerungen, die dieses Verhalten begleiten. Diese Äußerungen können dem Verhalten vorhergehen, sie können ihm nachfolgen, es übersetzen, es vorschreiben, es verstellen, es rechtfertigen usw. Andererseits wollte ich mich auch von dem absetzen, was man eine Geschichte der Vorstellungen oder der Vorstellungssysteme nennen könnte, d.h. eine Geschichte, die zwei Ziele haben könnte bzw. tatsächlich haben sollte. Das eine wäre die Analyse der Vorstellungsfunktionen. Unter >Analyse der Vorstellungsfunktionen< verstehe ich die Analyse der Rolle, die diese Vorstellungen spielen können, und zwar entweder im Hinblick auf das vorgestellte Objekt oder im Hinblick auf das vorstellende Subjekt - sagen wir, eine Art Ideologieanalyse. Der andere Pol einer möglichen Analyse der Vorstellungen scheint mir dann in der Analyse der Vorstellungswerte eines Vorstellungssystems zu bestehen, d.h. in der Analyse der Vorstellungen in Abhängigkeit von einer Erkenntnis - d.h. eines Erkenntnisinhalts oder einer Regel, einer Erkenntnisform -, betrachtet als Wahr- 
heitskriterium oder zumindest als Anhaltspunkt für die Wahrheit, auf die hin man den Vorstellungswert dieses oder jenes Cedankensystems, verstanden als System von Vorstellungen eines bestimmten Gegenstands, bestimmen kann. Zwischen diesen beiden Möglichkeiten also, zwischen diesen beiden Themen (der Mentalitätsgeschichte und der Vorstellungsgeschichte), habe ich versucht, eine Geschichte des Denkens zu schreiben. Und mit >Denken was man die Brennpunkte der Erfahrung nennen könnte, an denen sich die einen gegenüber den anderen artikulieren: An erster Stelle stehen hier die Formen eines möglichen Wissens; zweitens die normativen Verhaltensmatrizen der Individuen; und schließlich virtuelle Existenzmodi für mögliche Subjekte. Diese drei Elemente - Formen des möglichen Wissens, normative Verhaltensmatrizen, virtuelle Existenzmodi möglicher Subjekte -, das sind die drei Dinge oder vielmehr ist es die Gliederung dieser drei Dinge, die man >Brennpunkte der Erfahrung ^nennen kann.«(Foucault [1983] 2009: 14f [Herv. i. O.])

Am Lehrstuhl für die Geschichte des Denkens am Collége de France hat Foucault jahrelang daran gearbeitet, die Analyse unseres historisch wie gesellschaftlich bedingten Denkens zu ermöglichen. Seine Geschichtsschreibung musste er jedoch von zwei bereits etablierten Analyse- und Schreibvorgängen abgrenzen - der sog. Mentalitätsgeschichte (franz. l'histoire des mentalités) und der sog. Vorstellungsgeschichte (franz. l'histoire des représentations).

\subsection{Die Mentalitätsgeschichte}

Die erstere, betrieben vor allem in den 1960er Jahren in Frankreich, richtete ihren Blick auf die Untersuchung der Mentalitäten. ${ }^{1}$ Diese lassen sich als »auf der Grundlage bestimmter gesellschaftlicher Verhältnisse entstehenden Einstellungen, die sich zu habituell geprägten Denk-, Urteils- und Handlungsstrukturen verdichten« (Schönhuth 2006) beschreiben. Mehr als nur für das Denken, interessierte sich diese Vorgehensweise für das wahre Empfinden und für die tatsächlichen Verhaltensweisen der Menschen:

In Frankreich wurde diese historische Methodologie von der sog. Annales Schule vertreten und entwickelt (vertreten von bspw. Marc Bloch, Lucien Febvre, Fernand Braudel u.a.). Wie Mirjana Gross schreibt, kritisierte Foucault die Mentalitätsgeschichte und die Vertreter der Annales Schule, »weil sie in ihren Forschungen mehr die Gesellschaft als die geistigen Erscheinungen berücksichtigten. Als Kritiker der Mentalitätsgeschichte stand Foucault im Cegensatz zu Febvres Auffassung der Mentalität und ihrer späteren Aufarbeitung. Die Mentalitätsgeschichte der >Annales` befaßte sich mit Kategorien, die sie für allgemein hielt (Unglauben, Liebe, Furcht), während Foucault sie als vorläufige, diskontinuierliche Kategorien darstellte, die durch Praxis und Diskurs in einer bestimmten Zeit und einem Raum entstanden waren.« (Gross 1998: 389 [Herv. i. O.]) 
»/hr Anliegen war nicht mehr, das Handeln irgendwie als gegeben oder fertig unterstellten Menschen zu beschreiben und zu erklären, sondern den Prozess der Menschwerdung selbst, oder richtiger: die Prozesse, durch die Menschen zu dem wurden, was sie jeweils waren, zu ergründen. Damit verband sich die Frage, welchen Anteil der Mensch als denkendes, fühlendes, wünschendes Wesen an diesen Prozessen genommen hatte, also die Frage, wie man früher gesagt hätte, nach dem >subjektiven Faktor in der Ceschichte.«(Raulff 1987: 7 [Herv. i. O.])

Anders als bei dem ideengeschichtlichen Vorgang, versuchte sie »die Schwelle des Geschichtsfähigen nach >unten (a.a.O.: 15 [Herv. i. O.]) Mit dieser Verschiebung ändert sich auch die Auswahl von Analysematerialien, welche von nun an aus spezifisch aussagefähigen Quellen wie Gerichts- oder Kirchenakten, Diarien, Reiseliteratur, Manieren- und Predigtbüchern, Bildwerken und anderen ähnlichen Dokumenten bestehen (vgl. ebd.). Die Mentalitätsgeschichte bemühte sich also darum, eine Antwort auf die Frage zu bekommen, wie die Realität tatsächlich, bei den anonymen Massen, gedacht, empfunden und erlebt wird.

\subsection{Die Vorstellungsgeschichte}

Die zweite mögliche Geschichtsschreibung, die Foucault die Vorstellungsgeschichte nannte, interessierte sich für die Entwicklung der kollektiven Vorstellungen über gesellschaftliche, kulturelle oder politische Phänomene. Auf dem Gebiet der Politikwissenschaft repräsentiert diesen Vorgang die Ideengeschichte. Den Gegenstand einer solchen Geschichte bildet »die Entstehung und Entwicklung von politischen Ideen, worunter Muster, Schemata, Figuren, Bilder, Modelle, nicht zuletzt Begriffe fallen, in denen sich das allgemeine politische Denken bewegt." (Llanque 2008:3) Anders als bei der Mentalitätsgeschichte, liegen im Zentrum dieser Analyse »alle menschlichen Artefakte [...], die politisch relevante Ideen spiegeln.« (Ebd.) Deshalb ist auch ihr Material nicht anonym, sondern besteht aus öffentlichen Dokumenten, Gesetzestexten, Verfassungstexten, Bilderprogramme oder literarischen Texten und ihrer öffentlichen Diskussion (vgl. ebd.).

Mit dieser Art der Geschichtsschreibung entsteht ein Kontinuum an Wissen, das »immer wieder neu aufgenommen und verarbeitet wird, was im besten Falle neue Denkwege eröffnet und so künftiger Rezeption neues Material bietet, um zur anhaltenden, offenkundig nicht abzuschließenden Aufgabe beizutragen, die politische Selbstauslegung des Menschen in seiner Zeit zu orientieren.«(a.a.O.: 9) Damit lag die Aufgabe solcher Geschichtsschreibung darin, das spezialisierte Wissen, zu welchem nur wenige ausgebildete Individuen den Zugang hatten, als realitätsstiftende Grundlage zu beziehen und die Geschichte als Entwicklung und Fortsetzung dieses Wissensdiskurses vornehmlich zu interpretieren. Zwischen diesen beiden 
Möglichkeiten einer historischen Analyse versuchte Foucault eine sog. Geschichte des Denkens (franz. l'histoire de la pensée) zu platzieren.

\subsection{Die Geschichte des Denkens}

Den Analysevorgang einer Geschichte des Denkens hat Foucault mit der Problematisierung des Wahns begonnen und mit ihr lassen sich seine Grundzüge am besten erhellen. Was den Bereich dieser Analyse von den Bereichen der obengenannten Vorgänge unterscheidet, ist, dass dieser zuallererst geschaffen werden muss. Er ist nicht per se vorhanden, sondern muss zuerst als Analyseproblem sichtbar gemacht werden.

Wenn Foucault seine Analyse des Wahnsinns beginnt, so schreibt er, dass wir "von jener Geste des Einschnitts, jener eingenommenen Distanz, jener zwischen der Vernunft und dem, was sie nicht ist, hergestellten Leere sprechen [müssen], ohne uns je auf die Fülle zu stützen, die zu sein sie vorgibt.« (Foucault [1969] 1981: 8) Das heißt, die Dialektik des Wahnsinns und der Vernunft zu verfolgen und sie in ihrer stummen Sprache zu entziffern, dort, wo beide Begriffe noch nicht abgetrennt erscheinen: »Man muss in der Geschichte jenen Punkt Null der Geschichte des Wahnsinns wiederzufinden versuchen, an dem der Wahnsinn noch undifferenzierte Erfahrung, noch nicht durch eine Trennung gespaltene Erfahrung ist.« (a.a.O.: 7) Demnach war es für Foucault notwendig, auch den Gegenstand dieses Analysevorgangs anders zu definieren. Anstatt sich auf die tatsächliche Wahrnehmung und Empfindung der Realität (Mentalitätsgeschichte) oder auf das Formulieren kollektiver Vorstellungen (Vorstellungsgeschichte) zu konzentrieren, richtet Foucault den Blick auf die unausgesprochene, auf die fehlende Erfahrung:

»Die Fülle der Geschichte ist nur in dem leeren und zugleich bevölkerten Raum all jener Wörter ohne Sprache möglich, die einen tauben Lärm denjenigen hören lassen, der sein Ohr leiht, einen tauben Lärm von unterhalb der Ceschichte, das obstinate Gemurmel einer Sprache, die >von allein< spricht, ohne sprechendes Subjekt und ohne Gesprächspartner, auf sich selbst gehäuft, in der Gurgel geballt, und die noch zusammenbricht, bevor jegliche Formulierung erreicht ist, und ohne Aufsehen in das Schweigen zurückkehrt, aus dem sie sich nie befreit hat. Es ist die verkalkte Wurzel des Sinnes.«(a.a.O.: 12 [Herv. i. O.])

Außerdem war es für Foucault wichtig, eine spezifische Distanz zu dem analytischen Material zu gewinnen. Foucault wollte nämlich »außerhalb jeder Beziehung zu einer psychiatrischen $>$ Wahrheit $<$ jene Worte und Texte für sich sprechen lassen, die von unterhalb der Sprache stammen und die nicht dazu geschaffen waren, zu einer Rede zu werden.« (a.a.O.: 15 [Herv. i. O.]) Das heißt, dass das konstitutive Material für die Untersuchung nicht die offiziellen Texte, Diagnosen und Berichte einerseits, Notizhefte, Tagebücher oder Memoires andererseits waren, sondern die 
unausgesprochene Erfahrung, die in all den obengenannten Materialen zwar enthalten, aber nicht artikuliert ist. Und genau um das Reden-Lassen dieser Erfahrung bemüht sich Foucault in seiner Analytik. Dafür und für das Aufschreiben einer solchen Geschichte bedürfte es einer »ziemlich neutralen Sprache, die relativ frei von wissenschaftlicher Terminologie, sozialen oder moralischen Optionen war, damit sie so nahe wie möglich an jene primitiv miteinander verketteten Worte herankommen konnte und damit jene Distanz aufgehoben wurde, durch die sich der moderne Mensch gegen den Wahnsinn absichert.« (a.a.O.: 16)

In seiner Geschichte des Denkens wollte also Foucault eine Antwort auf die Frage erhalten, wie die Realität (noch) denkbar gemacht werden kann, und zeigen, wie viel unausgesprochenes und undifferenziertes Wissen einer historischen Erfahrung (noch) innewohnend ist. Diese Art der Geschichte ähnelt einer Mentalitätsgeschichte, indem sie möglichst nah an den Erfahrungen mit der Realität (durch Wahrnehmung, Handeln und Denken) operiert und sie nähert sich auch der Vorstellungsgeschichte, indem sie sich an der Entstehung des kollektiven Wissens abarbeitet. Doch sie distanziert sich von der ersteren durch ihren Anspruch, die Geschichte als nicht gegeben, sondern als kontingent zu schreiben. In dieser Geschichte stellen die Einstellungen der Menschen (die Mentalitäten) eine Schablone dar, an der die diskursiven Praktiken abgelesen werden könnten. Die Art und Weise des Denkens, Handelns und Empfindens lässt sich demnach als bedingt und gesellschaftlich produziert analysieren. Sie distanziert sich auch von der letzteren durch ihren Anspruch, eine "Archäologie des Schweigens« (a.a.O.: 8) zu schreiben, anstatt mit dem kollektiven Wissen (mit den Vorstellungen) in Dialog zu treten, dieses Wissen zu erweitern oder es zu vertiefen. Der Geschichte des Denkens geht es nicht darum, neue Erkenntnisse zu erschließen, sondern das Unausgesprochene, das Vergessene, das Ausgeschlossene oder das Verdrängte (wieder) ins Bewusstsein $\mathrm{zu}$ rücken und als eine verstummte Realität erscheinen zu lassen.

Mit der Geschichte des Denkens führt Foucault auch eine andere Art der Problemstellung bzw. Problematisierung in die wissenschaftliche Analyse ein, die er folgenderweise beschreibt:

»Problematisierung bedeutet nicht die Darstellung eines zuvor existierenden Objekts, genauso wenig aber auch die Erschaffung eines nicht existierenden Objekts durch den Diskurs. Die Gesamtheit der diskursiven oder nicht-diskursiven Praktiken lässt etwas in das Spiel des Wahren und des Falschen eintreten und konstituiert es als Objekt für das Denken (sei es in Form der moralischen Reflexion, der wissenschaftlichen Erkenntnis, der politischen Analyse usw.).«(Foucault [1984] 2005a: 826)

Damit beschrieb Foucault ein Analyseverfahren, das fragen wollte, »Wie und warum bestimmte Dinge (Verhalten, Erscheinungen, Prozesse) zum >Problem den« (Foucault [1983] 1996: 178 [Herv. i. O.]) und wie sie sich während der Geschichte 
so dargestellt und festgelegt haben: »Warum wurden zum Beispiel bestimmte Verhaltensformen als >Wahnsinn gekennzeichnet und klassifiziert, während ähnliche Formen in einem bestimmten historischen Augenblick völlig vernachlässigt wurden; dasselbe gilt für Verbrechen und Kriminalität, dieselbe Frage der Problematisierung gilt für die Sexualität.« (Ebd. [Herv. i. O.]) Diesem Vorgehen nach lassen sich auch weitere Phänomene, darunter auch das Präventionsdispositiv, als Ergebnisse langjähriger Transformationen entschlüsseln, die bis in die Gegenwart ihre Wirkungen haben. Und um ihre Konstruktion verstehen zu können, muss man »eine historische Analyse der Transformation des Diskurses vornehmen, ohne auf das Denken der Menschen, ihre Wahrnehmungsweisen, ihre Gewohnheiten, die Einflüsse, denen sie ausgesetzt waren usw. zu rekurrieren." (Foucault [1969] 2001: 982)

Für das Anliegen einer solchen Geschichte des Denkens als Analyse der Problematisierungen entwickelte Foucault drei Analyseachsen, drei, wie er sie nannte, Brennpunkte der Erfahrung, die ihm ermöglicht haben, sich einem spezifischen Problem zu nähern und ihm eine alternative Auslegung mit den daraus resultierenden gesellschaftspolitischen Konsequenzen anzubieten. Diese Brennpunkte bzw. Analyseachsen werden im Folgenden näher beschrieben.

\section{Foucaults Analyseinstrumente}

Bei seiner Vorgehensweise hat Foucault seine Analyseinstrumente stets anhand der Forschungsgegenstände entwickelt, die er zu analysieren beabsichtigte. Demnach bilden die drei Analyseachsen eher heuristische Kategorien, die das Analyseverfahren $\mathrm{zu}$ verdeutlichen helfen.

\subsection{Produktion des Wissens}

Foucaults erste Analyseachse konzentrierte sich auf die Produktion und Kanalisierung des Wissens, durch das die Ordnung in der Gesellschaft hergestellt wird. Beeinflusst von Jean Hyppolite und Louis Althusser, Friedrich Nietzsche, Pierre Klossowski, Maurice Blanchot und Georges Bataille (vgl. Foucault [1978] 2005b: 53; auch Kehm 1991: 33) war Foucault daran interessiert, die diskursive Natur des Wissens zu problematisieren und den Einfluss der Produktion von Sinnhaftigkeiten, Bedeutungen und Zuschreibungen auf die subjektive und kollektive Wahrnehmung $\mathrm{zu}$ untersuchen. Gerade in der Wissensproduktion wurzelt seiner Meinung nach das Problem, dass bestimmte Aussagen einen allgemeinen Geltungs- und Wahrheitsanspruch erheben: 